\title{
Existence of Solutions for Fractional Differential Equations with p-Laplacian Operator and Integral Boundary Conditions
}

\author{
Jingli Xie $(D)$ and Lijing Duan $(1)$ \\ College of Mathematics and Statistics, Jishou University, Jishou, Hunan 416000, China \\ Correspondence should be addressed to Lijing Duan; duanlijing1995@163.com
}

Received 10 February 2020; Revised 17 April 2020; Accepted 1 May 2020; Published 10 June 2020

Academic Editor: Liguang Wang

Copyright (C) 2020 Jingli Xie and Lijing Duan. This is an open access article distributed under the Creative Commons Attribution License, which permits unrestricted use, distribution, and reproduction in any medium, provided the original work is properly cited.

In this paper, we investigate a class of integral boundary value problems of fractional differential equations with a $p$-Laplacian operator. Existence of solutions is obtained by using the fixed point theorem, and an example is given to show the applicability of our main result.

\section{Introduction}

In this paper, we consider the nonlinear fractional differential equations with a $p$-Laplacian operator and integral boundary conditions

$$
\left\{\begin{array}{l}
{ }^{c} D_{0+}^{\beta}\left(\phi_{p}\left({ }^{c} D_{0+}^{\alpha} u(t)\right)\right)+f(t, u(t))=0, \quad t \in[0,1] \\
u(1)=\lambda \int_{0}^{1} u(s) d s, \quad u^{\prime}(1)=0 \\
{ }^{c} D_{0+}^{\alpha} u(1)=b^{c} D_{0+}^{\alpha} u(\xi),
\end{array}\right.
$$

where $1<\alpha \leq 2,0<\beta \leq 1,0<\xi, b, \lambda<1,{ }^{c} D_{0+}^{\alpha}$ and ${ }^{c} D_{0+}^{\beta}$ are the Caputo fractional derivative.

$\phi_{p}(s)=|s|^{p-2} s$ is the $p$-Laplacian operator such that $(1 / p)+(1 / q)=1, p>1$, and $\phi_{p}^{-1}(s)=\phi_{q}(s)$, and $f(t, u):[0$, $1] \times[0, \infty) \longrightarrow[0, \infty)$ is a given continuous function.

In recent years, boundary value problems of fractional differential equations have significantly been discussed by some researchers because fractional calculus theory and methods have been widely used in various fields of natural sciences and social sciences. In the field of physical mechanics, fractional calculus not only provides suitable mathematical tools for the study of soft matter but also provides new research ideas and plays an irreplaceable role in the modeling of soft matter [1-3]. Some nonlinear analysis tools such as coincidence degree theory $[4,5]$, upper and lower solution method [6-8], fixed point theorems [9-11], and variational methods [12-14] have been widely used to discuss existence of solutions for boundary value problems of fractional differential equations.

On the other hand, it is well known that differential equation models with $p$-Laplacian operators are often used to simulate practical problems such as tides caused by celestial gravity and elastic deformation of beams and rich results of fractional differential equations with a $p$-Laplacian operator have been obtained [15-18]. In particular, in [15], by using the fixed point theorem, Yan et al. studied the existence of solutions for boundary value problems of fractional differential equations with a $p$-Laplacian operator:

$$
\left\{\begin{array}{l}
-D_{0+}^{\alpha}\left(\phi_{p}\left(D_{0+}^{\beta} u(t)\right)\right)=f(t, u(t)), \\
u(0)=u(1)=u^{\prime}(0)=u^{\prime}(1), \\
D_{0+}^{\beta} u(0)=0, \quad D_{0+}^{\beta} u(1)=b D_{0+}^{\beta} u(\eta),
\end{array}\right.
$$

where $1<\alpha \leq 2,3<\beta \leq 4,0<\eta<1,0<b<\eta^{(1-\alpha) /(p-1)}$, (1/ $p)+(1 / q)=1,1<p, \phi_{p}^{-1}(s)=\phi_{q}(s), D_{0+}^{\alpha}, D_{0+}^{\beta}$ is the standard Riemann-Liouville derivative, and $f(t, u):(0,1) \times(0, \infty)$ $\longrightarrow[0, \infty)$ is a given countinuous function.

Moreover, during the last decade, the integral boundary value problem of fractional differential equations is also a 
hot issue for scholars and some good results have been achieved [19-23]. In [24], by using the method of the upper and lower solutions and Schauder's and Banach's fixed points theorem, Abdo et al. obtained the existence and uniqueness of a positive solution of the fractional differential equations with integral boundary equations:

$$
\left\{\begin{array}{l}
{ }^{c} D_{0+}^{\alpha} u(t)=f(t, u(t)), \quad t \in(0,1] \\
u(0)=\lambda \int_{0}^{1} u(s) d s+d
\end{array}\right.
$$

where $0<\alpha \leq 1, \lambda \geq 0, d>0,{ }^{c} D_{0+}^{\alpha}$ is the standard Caputo derivative, and $f:[0,1] \times[0, \infty) \longrightarrow[0, \infty)$ is a given countinuous function.

In [25], Bai and Qiu discuss the existence of positive solutions for boundary value problems of fractional differential equations:

$$
\left\{\begin{array}{l}
{ }^{c} D_{0+}^{\alpha} u(t)+f(t, u(t))=0, \quad t \in(0,1], \\
u(0)=u^{\prime}(1)=u^{\prime \prime}(0)=0,
\end{array}\right.
$$

where $2<\alpha \leq 3, f \in C([0,1] \times[0,+\infty),[0,+\infty))$, and ${ }^{c} D_{0+}^{\alpha}$ is the standard Caputo derivative.

Motivated by the works mentioned above, we concentrate on the solutions for the nonlinear fractional differential equation (1). We obtain the existence result of the fractional differential equations with integral boundary equations by using the Schauder fixed point theorem and other mathematical analysis techniques.

The rest of this paper is organized as follows. In Section 2, we give some notations and lemmas. Section 3 is devoted to study existence of solutions for boundary value problems of fractional differential equations. Finally, we provide an example to illustrate our results.

\section{Preliminaries}

In the section, we present some definitions and lemmas, which are required for building our theorems.
Definition 1 (see [1]). The fractional integral of order $\alpha(\alpha>0)$ of function $f:[0, \infty) \longrightarrow R$ is given by

$$
I_{0+}^{\alpha} f(t)=\frac{1}{\Gamma(\alpha)} \int_{0}^{t}(t-s)^{\alpha-1} f(s) d s,
$$

where $\Gamma(\alpha)$ is the Gamma function, provided the right side is pointwise defined on $(0,+\infty)$.

Definition 2 (see [2]). The Caputo fractional derivative of order $\alpha(\alpha>0)$ of function $f:[0, \infty) \longrightarrow R$ is given by

$$
{ }^{c} D^{\alpha} f(t)=\frac{1}{\Gamma(n-\alpha)} \int_{0}^{t}(t-s)^{n-\alpha-1} f^{(n)}(s) d s,
$$

where $t>0, n=[\alpha]+1, \Gamma(\alpha)$ is the Gamma function.

Lemma 3 (see [26]). For $\alpha>0$, the solution of fractional differential equation ${ }^{c} D_{0+}^{\alpha} u(t)=0$ is given by $u(t)=c_{0}+$ $c_{1} t+\cdots+c_{n-1} t^{n-1}, c_{i} \in R, i=0,1,2, \cdots, n-1, n=[\alpha]+1$, and $[\alpha]$ denotes the integer part of the real number $\alpha$.

Lemma 4 (see [1]). For $\alpha>0$, then (i) $I_{0+}^{\alpha}\left({ }^{c} D_{0+}^{\alpha} u(t)\right)=u(t)+$ $c_{0}+c_{1} t+\cdots+c_{n-1} t^{n-1}, \quad c_{i} \in R, \quad n=[\alpha]+1$ and (ii) ${ }^{c} D_{0+}^{\alpha} I_{0+}^{\alpha}$ $u(t)=u(t)$.

Lemma 5 (see [1]). Let $X$ be a Banach space and $\Omega \subset X$ a convex, closed, and bounded set. If $T: \Omega \longrightarrow \Omega$ is a continuous operator such that $T \Omega \subset X, T \Omega$ is relatively compact, then $T$ has at least one fixed point in $\Omega$.

Let $\phi_{p}\left({ }^{c} D_{0+}^{\alpha} u(t)\right)=v(t)$, then $v(1)=b^{p-1} v(\xi)$. We now consider the following equations:

$$
\left\{\begin{array}{l}
-{ }^{c} D_{0+}^{\beta} v(t)=y(t), \quad t \in[0,1], \\
v(1)=b^{p-1} v(\xi) .
\end{array}\right.
$$

Lemma 6. Lety $\in C[0,1]$, then (7) has a unique solution

$$
v(t)=\int_{0}^{1} H(t, \tau) y(\tau) d \tau,
$$

where

$$
H(t, \tau)=\left\{\begin{array}{lll}
\frac{(1-\tau)^{\beta-1}}{\left(1-b^{p-1}\right) \Gamma(\beta)}-\frac{(t-\tau)^{\beta-1}}{\Gamma(\beta)}, & 0 \leq \tau \leq t \leq 1, \quad \xi \leq \tau, \\
\frac{(1-\tau)^{\beta-1}}{\left(1-b^{p-1}\right) \Gamma(\beta)}-\frac{(t-\tau)^{\beta-1}}{\Gamma(\beta)}-\frac{b^{p-1}(\xi-\tau)^{\beta-1}}{\left(1-b^{p-1}\right) \Gamma(\beta)}, & 0 \leq \tau \leq t \leq 1, \quad \xi \leq \tau, \\
\frac{(1-\tau)^{\beta-1}}{\left(1-b^{p-1}\right) \Gamma(\beta)}, & 0 \leq t \leq \tau \leq 1, & \xi \leq \tau, \\
\frac{(1-\tau)^{\beta-1}}{\left(1-b^{p-1}\right) \Gamma(\beta)}-\frac{b^{p-1}(\xi-\tau)^{\beta-1}}{\left(1-b^{p-1}\right) \Gamma(\beta)}, & 0 \leq t \leq \tau \leq 1, & \xi \leq \tau .
\end{array}\right.
$$


Proof. Suppose $v$ satisfies boundary value problem (7), by (i) of Lemma 4 , we can obtain

$$
v(t)=-I_{0+}^{\beta} y(t)-c_{0}=-\frac{1}{\Gamma(\beta)} \int_{0}^{t}(t-s)^{\beta-1} y(s) d s-c_{0} .
$$

Using the boundary condition $v(1)=b^{p-1} v(\xi)$, we can obtain

$$
\begin{aligned}
c_{0}= & \frac{b^{p-1}}{\left(1-b^{p-1}\right) \Gamma(\beta)} \int_{0}^{\xi}(\xi-s)^{\beta-1} y(s) d s \\
& -\frac{1}{\left(1-b^{p-1}\right) \Gamma(\beta)} \int_{0}^{1}(1-s)^{\beta-1} y(s) d s .
\end{aligned}
$$

Thus,

$$
\begin{aligned}
v(t)= & -\frac{1}{\Gamma(\beta)} \int_{0}^{t}(t-s)^{\beta-1} y(s) d s \\
& -\frac{b^{p-1}}{\left(1-b^{p-1}\right) \Gamma(\beta)} \int_{0}^{\xi}(\xi-s)^{\beta-1} y(s) d s \\
& +\frac{1}{\left(1-b^{p-1}\right) \Gamma(\beta)} \int_{0}^{1}(1-s)^{\beta-1} y(s) d s=\int_{0}^{1} H(t, s) y(s) d s .
\end{aligned}
$$

From the above analysis, the equation

$$
\left\{\begin{array}{l}
{ }^{c} D_{0^{+}}^{\beta}\left(\phi_{p}\left({ }^{c} D_{0^{+}}^{\alpha} u(t)\right)\right)+y(t)=0 \\
u(1)=\lambda \int_{0}^{1} u(s) d s, \quad u^{\prime}(1)=0 \\
{ }^{c} D_{0^{+}}^{\alpha} u(1)=b^{c} D_{0^{+}}^{\alpha} u(\xi)
\end{array}\right.
$$

is equivalent to

$$
\left\{\begin{array}{l}
{ }^{c} D_{0^{+}}^{\alpha} u(t)=\phi_{q}\left(\int_{0}^{1} H(t, \tau) y(\tau) d \tau\right), \quad 0<t<1, \\
u^{\prime}(1)=0, \quad u(1)=\lambda \int_{0}^{1} u(s) d s .
\end{array}\right.
$$

Lemma 7. Lety $\in C[0,1]$. Then (14) has a unique solution:

$$
\begin{aligned}
u(t)= & \left\{\frac{1}{\Gamma(\alpha)} \int_{0}^{t}(t-s)^{\alpha-1} \phi_{q}\left(\int_{0}^{1} H(s, \tau) y(\tau) d \tau\right) d s\right. \\
& -\frac{1}{\Gamma(\alpha)} \int_{0}^{1}(1-s)^{\alpha-1} \phi_{q}\left(\int_{0}^{1} H(s, \tau) y(\tau) d \tau\right) d s \\
& +\frac{1}{\Gamma(\alpha-1)} \int_{0}^{1}(1-s)^{\alpha-2} \phi_{q}\left(\int_{0}^{1} H(s, \tau) y(\tau) d \tau\right) d s \\
& -\frac{1}{\Gamma(\alpha-1)} \int_{0}^{1} t(1-s)^{\alpha-2} \phi_{q}\left(\int_{0}^{1} H(s, \tau) y(\tau) d \tau\right) d s+\lambda A,
\end{aligned}
$$

where

$$
\begin{aligned}
A= & \frac{1}{1-\lambda}\left[\frac{1}{\Gamma(\alpha+1)} \int_{0}^{1}(1-s)^{\alpha} \phi_{q}\left(\int_{0}^{1} H(s, \tau) y(\tau) d \tau\right) d s\right. \\
& -\frac{1}{\Gamma(\alpha)} \int_{0}^{1}(1-s)^{\alpha-1} \phi_{q}\left(\int_{0}^{1} H(s, \tau) y(\tau) d \tau\right) d s \\
& \left.+\frac{1}{2 \Gamma(\alpha-1)} \int_{0}^{1}(1-s)^{\alpha-2} \phi_{q}\left(\int_{0}^{1} H(s, \tau) y(\tau) d \tau\right) d s\right] .
\end{aligned}
$$

Proof. By (i) of Lemma (4), we can obtain $u(t)=(1 / \Gamma(\alpha))$ $\int_{0}^{t}(t-s)^{\alpha-1} \phi_{q}\left(\int_{0}^{1} H(s, \tau) y(\tau) d \tau\right) d s-c_{1}-c_{2} t$. Then, $u^{\prime}(t)=$ $(1 / \Gamma(\alpha-1)) \int_{0}^{t}(t-s)^{\alpha-2} \phi_{q}\left(\int_{0}^{1} H(s, \tau) y(\tau) d \tau\right) d s-c_{2}$, using the boundary condition $u^{\prime}(1)=0$, we can obtain

$$
c_{2}=\frac{1}{\Gamma(\alpha-1)} \int_{0}^{1}(1-s)^{\alpha-2} \phi_{q}\left(\int_{0}^{1} H(s, \tau) y(\tau) d \tau\right) d s .
$$

Another, because

$$
\begin{aligned}
& u(1)=\lambda \int_{0}^{1} u(s) d s \\
& u(1)=\frac{1}{\Gamma(\alpha)} \int_{0}^{1}(1-s)^{\alpha-1} \phi_{q}\left(\int_{0}^{1} H(s, \tau) y(\tau) d \tau\right) d s-c_{1}-c_{2}
\end{aligned}
$$

we have

$$
\begin{aligned}
c_{1}= & \frac{1}{\Gamma(\alpha)} \int_{0}^{1}(1-s)^{\alpha-1} \phi_{q}\left(\int_{0}^{1} H(s, \tau) y(\tau) d \tau\right) d s \\
& -\frac{1}{\Gamma(\alpha-1)} \int_{0}^{1}(1-s)^{\alpha-2} \phi_{q}\left(\int_{0}^{1} H(s, \tau) y(\tau) d \tau\right) d s-\lambda \int_{0}^{1} u(s) d s .
\end{aligned}
$$

Now, we express $\int_{0}^{1} u(s) d s$, let

$$
\begin{aligned}
A= & \int_{0}^{1} u(t) d t=\int_{0}^{1} \int_{0}^{t} \frac{(t-s)^{\alpha-1}}{\Gamma(\alpha)} \phi_{q}\left(\int_{0}^{1} H(s, \tau) y(\tau) d \tau\right) d s d t \\
& -\int_{0}^{1} \int_{0}^{1} \frac{(1-s)^{\alpha-1}}{\Gamma(\alpha)} \phi_{q}\left(\int_{0}^{1} H(s, \tau) y(\tau) d \tau\right) d s d t \\
& +\int_{0}^{1} \int_{0}^{1} \frac{(1-s)^{\alpha-2}}{\Gamma(\alpha-1)} \phi_{q}\left(\int_{0}^{1} H(s, \tau) y(\tau) d \tau\right) d s d t \\
& -\int_{0}^{1} \int_{0}^{1} \frac{t(1-s)^{\alpha-2}}{\Gamma(\alpha-1)} \phi_{q}\left(\int_{0}^{1} H(s, \tau) y(\tau) d \tau\right) d s d t \\
& +\int_{0}^{1} \lambda A d t=\int_{0}^{1} \frac{(1-s)^{\alpha}}{\Gamma(\alpha+1)} \phi_{q}\left(\int_{0}^{1} H(s, \tau) y(\tau) d \tau\right) d s \\
& -\int_{0}^{1} \frac{(1-s)^{\alpha-1}}{\Gamma(\alpha)} \phi_{q}\left(\int_{0}^{1} H(s, \tau) y(\tau) d \tau\right) d s \\
& +\int_{0}^{1} \frac{(1-s)^{\alpha-2}}{\Gamma(\alpha-1)} \phi_{q}\left(\int_{0}^{1} H(s, \tau) y(\tau) d \tau\right) d s \\
& -\int_{0}^{1} \frac{(1-s)^{\alpha-2}}{2 \Gamma(\alpha-1)} \phi_{q}\left(\int_{0}^{1} H(s, \tau) y(\tau) d \tau\right) d s+\lambda A
\end{aligned}
$$


We obtain

$$
\begin{aligned}
A= & \frac{1}{1-\lambda}\left[\frac{1}{\Gamma(\alpha+1)} \int_{0}^{1}(1-s)^{\alpha} \phi_{q}\left(\int_{0}^{1} H(s, \tau) y(\tau) d \tau\right) d s\right. \\
& -\frac{1}{\Gamma(\alpha)} \int_{0}^{1}(1-s)^{\alpha-1} \phi_{q}\left(\int_{0}^{1} H(s, \tau) y(\tau) d \tau\right) d s \\
& \left.+\frac{1}{2 \Gamma(\alpha-1)} \int_{0}^{1}(1-s)^{\alpha-2} \phi_{q}\left(\int_{0}^{1} H(s, \tau) y(\tau) d \tau\right) d s\right]
\end{aligned}
$$

Therefore,

$$
\begin{aligned}
u(t)= & \frac{1}{\Gamma(\alpha)} \int_{0}^{t}(t-s)^{\alpha-1} \phi_{q}\left(\int_{0}^{1} H(s, \tau) y(\tau) d \tau\right) d s \\
& -\frac{1}{\Gamma(\alpha)} \int_{0}^{1}(1-s)^{\alpha-1} \phi_{q}\left(\int_{0}^{1} H(s, \tau) y(\tau) d \tau\right) d s \\
& +\frac{1}{\Gamma(\alpha-1)} \int_{0}^{1}(1-s)^{\alpha-2} \phi_{q}\left(\int_{0}^{1} H(s, \tau) y(\tau) d \tau\right) d s \\
& -\frac{1}{\Gamma(\alpha-1)} \int_{0}^{1} t(1-s)^{\alpha-2} \phi_{q}\left(\int_{0}^{1} H(s, \tau) y(\tau) d \tau\right) d s+\lambda A
\end{aligned}
$$

Reverse, if

$$
\begin{aligned}
u(t)= & \frac{1}{\Gamma(\alpha)} \int_{0}^{t}(t-s)^{\alpha-1} \phi_{q}\left(\int_{0}^{1} H(s, \tau) y(\tau) d \tau\right) d s \\
& -\frac{1}{\Gamma(\alpha)} \int_{0}^{1}(1-s)^{\alpha-1} \phi_{q}\left(\int_{0}^{1} H(s, \tau) y(\tau) d \tau\right) d s \\
& +\frac{1}{\Gamma(\alpha-1)} \int_{0}^{1}(1-s)^{\alpha-2} \phi_{q}\left(\int_{0}^{1} H(s, \tau) y(\tau) d \tau\right) d s \\
& -\frac{1}{\Gamma(\alpha-1)} \int_{0}^{1} t(1-s)^{\alpha-2} \phi_{q}\left(\int_{0}^{1} H(s, \tau) y(\tau) d \tau\right) d s+\lambda A
\end{aligned}
$$

by (ii) of Lemma (4), we can obtain that $u(t)$ is a solution of (14).

The proof is completed.

Lemma 8. The functions $H$ is continuous on $[0,1] \times[0,1]$ and has the following properties:

(1) $H(t, \tau) \leq H(\tau, \tau), \quad t, \tau \in[0,1]$

(2) $\int_{0}^{1} H(\tau, \tau) d \tau \leq\left(1 /\left(1-b^{p-1}\right) \Gamma(\beta+1)\right)$

Proof. (1) For any $t, \tau \in[0,1]$, by (9), it is obvious that $H$ $(t, \tau) \leq H(\tau, \tau)$. (2) For any $t, \tau \in[0,1]$, by (9), we conclude that

$$
H(\tau, \tau) \leq \frac{(1-\tau)^{\beta-1}}{\left(1-b^{p-1}\right) \Gamma(\beta)}
$$

Therefore,

$$
\int_{0}^{1} H(\tau, \tau) d \tau \leq \frac{1}{\left(1-b^{p-1}\right) \Gamma(\beta+1)}
$$

This completes the proof.

\section{Main Results}

In this section, we will show the existence results for boundary value problem (1) by the Schauder fixed point theorem.

Let $I=[0,1], \quad U=\{u(t) \mid u(t) \in C(I)\}$, and definite the $\operatorname{norm}\|u\|=\max _{t \in[0,1]}|u(t)|,(U,\|\bullet\|)$ is a Banach space.

Theorem 9. Assume that the following conditions $\left(H_{1}\right)$ and $\left(\mathrm{H}_{2}\right)$ are satisfied:

$\left(H_{1}\right) f(t, u):[0,1] \times[0, \infty) \longrightarrow[0, \infty)$ is continuous

$\left(H_{2}\right)$ There exists a constant $k>0$, satisfying $f(t, u) \leq L \phi_{p}$ $(u), t \in[0,1],\|u\| \leq k$, where

$$
0<L \leq\left[\phi_{p}\left(\frac{2(1-\lambda) \Gamma(\alpha+2)}{(2-\lambda)\left(\alpha^{2}+\alpha\right)}\right)\left(\frac{1}{\left(1-b^{p-1}\right) \Gamma(\beta+1)}\right)^{-1}\right]
$$

then the problem (1) has at least one solution.

Proof. Let $P=\{u(t) \mid\|u(t)\| \leq k, t \in[0,1]\}$; thus, $P \subset U$ is convex, bounded, and closed.

Define an operator $T: P \longrightarrow U$ by

$$
\begin{aligned}
T u(t)= & \frac{1}{\Gamma(\alpha)} \int_{0}^{t}(t-s)^{\alpha-1} \phi_{q}\left(\int_{0}^{1} H(s, \tau) f(\tau, u(\tau)) d \tau\right) d s \\
& -\frac{1}{\Gamma(\alpha)} \int_{0}^{1}(1-s)^{\alpha-1} \phi_{q}\left(\int_{0}^{1} H(s, \tau) f(\tau, u(\tau)) d \tau\right) d s \\
& +\frac{1}{\Gamma(\alpha-1)} \int_{0}^{1}(1-s)^{\alpha-2} \phi_{q}\left(\int_{0}^{1} H(s, \tau) f(\tau, u(\tau)) d \tau\right) d s \\
& -\frac{1}{\Gamma(\alpha-1)} \int_{0}^{1} t(1-s)^{\alpha-2} \phi_{q}\left(\int_{0}^{1} H(s, \tau) f(\tau, u(\tau)) d \tau\right) d s \\
& +\frac{\lambda}{1-\lambda}\left[\frac{1}{\Gamma(\alpha+1)} \int_{0}^{1}(1-s)^{\alpha} \phi_{q}\left(\int_{0}^{1} H(s, \tau) f(\tau, u(\tau)) d \tau\right) d s\right. \\
& -\frac{1}{\Gamma(\alpha)} \int_{0}^{1}(1-s)^{\alpha-1} \phi_{q}\left(\int_{0}^{1} H(s, \tau) f(\tau, u(\tau)) d \tau\right) d s \\
& \left.+\frac{1}{2 \Gamma(\alpha-1)} \int_{0}^{1}(1-s)^{\alpha-2} \phi_{q}\left(\int_{0}^{1} H(s, \tau) f(\tau, u(\tau)) d \tau\right) d s\right] .
\end{aligned}
$$

For any $u \in P$, then by $\left(H_{2}\right)$, we have

$$
f(t, u) \leq L \phi_{p}(u) \leq L \phi_{p}(k) .
$$


By Lemma (8), we conclude that

$$
\begin{aligned}
& |T u(t)| \leq \mid \frac{1}{\Gamma(\alpha)} \int_{0}^{t}(t-s)^{\alpha-1} \phi_{q}\left(\int_{0}^{1} H(\tau, \tau) f(\tau, u(\tau)) d \tau\right) d s \\
& -\frac{1}{\Gamma(\alpha)} \int_{0}^{1}(1-s)^{\alpha-1} \phi_{q}\left(\int_{0}^{1} H(\tau, \tau) f(\tau, u(\tau)) d \tau\right) d s \\
& +\frac{1}{\Gamma(\alpha-1)} \int_{0}^{1}(1-s)^{\alpha-2} \phi_{q}\left(\int_{0}^{1} H(\tau, \tau) f(\tau, u(\tau)) d \tau\right) d s \\
& -\frac{1}{\Gamma(\alpha-1)} \int_{0}^{1} t(1-s)^{\alpha-2} \phi_{q}\left(\int_{0}^{1} H(\tau, \tau) f(\tau, u(\tau)) d \tau\right) d s \\
& +\frac{\lambda}{1-\lambda}\left[\frac{1}{\Gamma(\alpha+1)} \int_{0}^{1}(1-s)^{\alpha} \phi_{q}\left(\int_{0}^{1} H(\tau, \tau) f(\tau, u(\tau)) d \tau\right) d s\right. \\
& -\frac{1}{\Gamma(\alpha)} \int_{0}^{1}(1-s)^{\alpha-1} \phi_{q}\left(\int_{0}^{1} H(\tau, \tau) f(\tau, u(\tau)) d \tau\right) d s \\
& \left.+\frac{1}{2 \Gamma(\alpha-1)} \int_{0}^{1}(1-s)^{\alpha-2} \phi_{q}\left(\int_{0}^{1} H(\tau, \tau) f(\tau, u(\tau)) d \tau\right) d s\right] \mid \\
& =\mid \frac{t^{\alpha}}{\Gamma(\alpha+1)} \phi_{q}\left(\int_{0}^{1} H(\tau, \tau) f(\tau, u(\tau)) d \tau\right) \\
& -\frac{1}{\Gamma(\alpha+1)} \phi_{q}\left(\int_{0}^{1} H(\tau, \tau) f(\tau, u(\tau)) d \tau\right) \\
& +\frac{1}{\Gamma(\alpha)} \phi_{q}\left(\int_{0}^{1} H(\tau, \tau) f(\tau, u(\tau)) d \tau\right) \\
& -\frac{t}{\Gamma(\alpha)} \phi_{q}\left(\int_{0}^{1} H(\tau, \tau) f(\tau, u(\tau)) d \tau\right) \\
& +\frac{\lambda}{1-\lambda}\left[\frac{1}{\Gamma(\alpha+2)} \phi_{q}\left(\int_{0}^{1} H(\tau, \tau) f(\tau, u(\tau)) d \tau\right)\right. \\
& -\frac{1}{\Gamma(\alpha+1)} \phi_{q}\left(\int_{0}^{1} H(\tau, \tau) f(\tau, u(\tau)) d \tau\right) \\
& \left.+\frac{1}{2 \Gamma(\alpha)} \phi_{q}\left(\int_{0}^{1} H(\tau, \tau) f(\tau, u(\tau)) d \tau\right)\right] \mid \\
& \leq \mid \frac{1}{\Gamma(\alpha+1)} \phi_{q}\left(\int_{0}^{1} H(\tau, \tau) f(\tau, u(\tau)) d \tau\right) \\
& -\frac{1}{\Gamma(\alpha+1)} \phi_{q}\left(\int_{0}^{1} H(\tau, \tau) f(\tau, u(\tau)) d \tau\right) \\
& +\frac{1}{\Gamma(\alpha)} \phi_{q}\left(\int_{0}^{1} H(\tau, \tau) f(\tau, u(\tau)) d \tau\right) \\
& +\frac{\lambda}{1-\lambda}\left[\frac{1}{\Gamma(\alpha+2)} \phi_{q}\left(\int_{0}^{1} H(\tau, \tau) f(\tau, u(\tau)) d \tau\right)\right. \\
& -\frac{1}{\Gamma(\alpha+1)} \phi_{q}\left(\int_{0}^{1} H(\tau, \tau) f(\tau, u(\tau)) d \tau\right) \\
& \left.+\frac{1}{2 \Gamma(\alpha)} \phi_{q}\left(\int_{0}^{1} H(\tau, \tau) f(\tau, u(\tau)) d \tau\right)\right] \mid \\
& =\left|\frac{2 \alpha^{2}+2 \alpha-\lambda \alpha^{2}-3 \lambda \alpha}{2(1-\lambda) \Gamma(\alpha+2)} \phi_{q}\left(\int_{0}^{1} H(\tau, \tau) f(\tau, u(\tau)) d \tau\right)\right| \\
& \leq\left|\frac{(2-\lambda)\left(\alpha^{2}+\alpha\right)}{2(1-\lambda) \Gamma(\alpha+2)} \phi_{q}\left(\int_{0}^{1} H(\tau, \tau) f(\tau, u(\tau)) d \tau\right)\right| \\
& \leq\left|\frac{(2-\lambda)\left(\alpha^{2}+\alpha\right)}{2(1-\lambda) \Gamma(\alpha+2)} \phi_{q}\left(\int_{0}^{1} H(\tau, \tau) L \phi_{p}(u(\tau)) d \tau\right)\right| \\
& \left.\leq \mid \frac{(2-\lambda)\left(\alpha^{2}+\alpha\right)}{2(1-\lambda) \Gamma(\alpha+2)} \phi_{q}\left(\int_{0}^{1} H(\tau, \tau) L \phi_{p}(k)\right) d \tau\right) \mid \\
& \leq \frac{(2-\lambda)\left(\alpha^{2}+\alpha\right)}{2(1-\lambda) \Gamma(\alpha+2)}\left|\phi_{q}\left(\int_{0}^{1} H(\tau, \tau) d \tau\right)\right| \phi_{q}(L) k \\
& \leq \frac{(2-\lambda)\left(\alpha^{2}+\alpha\right)}{2(1-\lambda) \Gamma(\alpha+2)}\left|\phi_{q}\left(\frac{1}{\left(1-b^{p-1}\right) \Gamma(\beta+1)}\right)\right| \phi_{q}(L) k \leq k .
\end{aligned}
$$

Thus, $T(P) \subseteq P$. By $\left(H_{2}\right)$, we have

$$
\begin{aligned}
& \left|\phi_{q}\left(\int_{0}^{1} H(s, \tau) f(\tau, u(\tau)) d \tau\right) d s\right| \\
& \quad \leq\left|\phi_{q}\left(\frac{L \phi_{p}(k)}{\left(1-b^{p-1}\right) \Gamma(\beta+1)}\right)\right| \leq\left(\frac{L \phi_{p}(k)}{\left(1-b^{p-1}\right) \Gamma(\beta+1)}\right)^{q-1} .
\end{aligned}
$$

We express

$$
M:=\left(\frac{L \phi_{p}(k)}{\left(1-b^{p-1}\right) \Gamma(\beta+1)}\right)^{q-1}
$$

For each $t_{1}, t_{2} \in[0,1], t_{1}<t_{2}$, we get

$$
\begin{array}{rl}
\mid T & u\left(t_{2}\right)-T u\left(t_{1}\right)|=|\left(\frac{1}{\Gamma(\alpha)} \int_{0}^{t_{2}}\left(t_{2}-s\right)^{\alpha-1} \phi_{q}\left(\int_{0}^{1} H(s, \tau) f(\tau, u(\tau)) d \tau\right) d s\right. \\
& \left.-\frac{1}{\Gamma(\alpha)} \int_{0}^{t_{1}}\left(t_{1}-s\right)^{\alpha-1} \phi_{q}\left(\int_{0}^{1} H(s, \tau) f(\tau, u(\tau)) d \tau\right) d s\right) \\
& -\left(\frac{1}{\Gamma(\alpha-1)} \int_{0}^{t} t_{2}(1-s)^{\alpha-2} \phi_{q}\left(\int_{0}^{1} H(s, \tau) f(\tau, u(\tau)) d \tau\right) d s\right. \\
& \left.-\frac{1}{\Gamma(\alpha-1)} \int_{0}^{1} t_{1}(1-s)^{\alpha-2} \phi_{q}\left(\int_{0}^{1} H(s, \tau) f(\tau, u(\tau)) d \tau\right) d s\right) \mid \\
& \leq\left(\frac{L \phi_{p}(k)}{\left(1-b^{p-1}\right) \Gamma(\beta+1)}\right)^{q-1}\left|\frac{t_{2}^{\alpha}-t_{1}^{\alpha}}{\Gamma(\alpha+1)}-\frac{t_{2}-t_{1}}{\Gamma(\alpha)}\right| \\
& \leq M\left|\frac{\left(t_{2}^{\alpha-1}+t_{2}^{\alpha-2} t_{1}+t_{2}^{\alpha-3} t_{1}^{2}+\cdots+t_{2} t_{1}^{\alpha-2}+t_{1}^{\alpha-1}\right)}{\Gamma(\alpha+1)}-\frac{1}{\Gamma(\alpha)}\right|\left|t_{2}-t_{1}\right| .
\end{array}
$$

As $t_{2} \longrightarrow t_{1}$, the right-hand side of the previous inequality is independent of $u$ and tends to zero; thus, $T(P)$ is equicontinuous. From the Arzela-Ascoli Theorem, $T$ is compact. Applying Schauder's fixed point theorem, $T$ has at least one fixed point $u \in P$. Therefore, the problem (1) has at least one positive solution $u$ in $P$.

\section{Applications}

In this section, we will give an example to illustrate our main results.

Example 1. Consider the following equation:

$$
\left\{\begin{array}{l}
{ }^{c} D_{0^{+}}^{1 / 2}\left(\phi_{4 / 3}\left({ }^{c} D_{0^{+}}^{3 / 2} u(t)\right)\right)=\frac{\phi_{p}|u(t)|}{(t+5)^{2}}, \quad t \in(0,1), \\
u(1)=\frac{1}{2} \int_{0}^{1} u(s) d s, u^{\prime}(1)=0, \quad{ }^{c} D_{0^{+}}^{\alpha} u(1)=\frac{1}{2}{ }^{c} D_{0^{+}}^{\alpha} u\left(\frac{1}{2}\right),
\end{array}\right.
$$

where $\beta=1 / 2, \alpha=3 / 2, p=4 / 3, q=4, \lambda=\xi=b=1 / 2$, and $f(t, u)=\phi_{p}|u(t)| /(t+5)^{2}$. Since $f$ is continuous and

$$
|f(t, u)| \leq \frac{1}{25} \phi_{p}|u(t)|
$$


for $(t, u) \in[0,1] \times[0, \infty)$, we obtain

$$
L=\frac{1}{25}
$$

Then,

$$
\left[\phi_{p}\left(\frac{2(1-\lambda) \Gamma(\alpha+2)}{(2-\lambda)\left(\alpha^{2}+\alpha\right)}\right)\left(\frac{1}{\left(1-b^{p-1}\right) \Gamma(\beta+1)}\right)^{-1}\right] \approx 0.1534
$$

It is obvious that

$L<\left[\phi_{p}\left(\frac{2(1-\lambda) \Gamma(\alpha+2)}{(2-\lambda)\left(\alpha^{2}+\alpha\right)}\right)\left(\frac{1}{\left(1-b^{p-1}\right) \Gamma(\beta+1)}\right)^{-1}\right] \approx 0.1534$

By Theorem (9), we conclude that the problem (34) has at least one solution.

\section{Data Availability}

No data were used to support this study.

\section{Conflicts of Interest}

The authors declare that they have no competing interests.

\section{Authors' Contributions}

Both authors made an equal contribution.

\section{Acknowledgments}

This research is funded by the National Natural Science Foundation of China (No:11661037) and Scientific Research Fund of Jishou University (No:Jdy19004).

\section{References}

[1] A. Kilbas, H. Srivastava, and J. Trujillo, Theory and Applications of Fractional Differential Equations, Elsevier, The Netherlands, 2006.

[2] I. Prodlubny, Fractional Differential Equations, Academic Press, New York, 1999.

[3] D. R. Smart, Fixed Point Theorems, Cup Archive, 1980.

[4] X. Tang, "Existence of solutions of four-point boundary value problems for fractional differential equations at resonance," Journal of Applied Mathematics and Computing, vol. 51, no. 1-2, pp. 145-160, 2016.

[5] X. Tang, C. Yan, and Q. Liu, "Existence of solutions of twopoint boundary value problems for fractional $p$-Laplace differential equations at resonance," Journal of Applied Mathematics and Computing, vol. 41, no. 1-2, pp. 119-131, 2013.

[6] A. Chidouh, A. Guezane-Lakoud, and R. Bebbouchi, "Positive solutions of the fractional relaxation equation using lower and upper solutions," Vietnam Journal of Mathematics, vol. 44, no. 4, pp. 739-748, 2016.
[7] X. Liu, M. Jia, and W. Ge, "The method of lower and upper solutions for mixed fractional four-point boundary value problem with $p$-Laplacian operator," Applied Mathematics Letters, vol. 65, pp. 56-62, 2017.

[8] Y. Wei, Q. Song, and Z. Bai, "Existence and iterative method for some fourth order nonlinear boundary value problems," Applied Mathematics Letters, vol. 87, pp. 101-107, 2019.

[9] S. K. Ntouyas and J. Tariboon, "Fractional boundary value problems with multiple orders of fractional derivatives and integrals," Electronic Journal of Differential Equations, vol. 2017, no. 100, pp. 1-18, 2017.

[10] Y. Cui, W. Ma, X. Wang, and X. Su, "New uniqueness results for boundary value problem of fractional differential equation," Nonlinear Analysis: Modelling and Control, vol. 23, pp. 31-39, 2018.

[11] X. Zhang and Q. Zhong, "Triple positive solutions for nonlocal fractional differential equations with singularities both on time and space variables," Applied Mathematics Letters, vol. 80, pp. 12-19, 2018.

[12] Z. H. Zhang and J. Li, "Variational approach to solutions for a class of fractional boundary value problems," Electronic Journal of Qualitative Theory of Differential Equations, vol. 2015, no. 11, pp. 1-10, 2015.

[13] C. Torres, "Mountain pass solution for a fractional boundary value problem," Journal of Fractional Calculus and Applications, vol. 5, no. 1, pp. 1-10, 2014.

[14] F. Jiao and Y. Zhou, "Existence of solutions for a class of fractional boundary value problems via critical point theory," Computers and Mathematics with Applications, vol. 62, no. 3, pp. 1181-1199, 2011.

[15] F. Yan, M. Zuo, and X. Hao, "Positive solution for a fractional singular boundary value problem with $p$-Laplacian operator," Boundary Value Problems, vol. 2018, no. 1, Article ID 51, 2018.

[16] Y. Li, "Existence of positive solutions for fractional differential equation involving integral boundary conditions with $p$-Laplacian operator," Advances in Difference Equations, vol. 135, 2476 pages, 2017.

[17] J. J. Tan and C. Z. Cheng, "Existence of solutions of boundary value problems for fractional differential equations with $p$ Laplacian operator in Banach spaces," Numerical Functional Analysis and Optimization, vol. 38, no. 6, pp. 738-753, 2016.

[18] X. Dong, Z. Bai, and S. Zhang, "Positive solutions to boundary value problems of $p$-Laplacian with fractional derivative," Boundary Value Problems, vol. 2017, no. 1, Article ID 5, 2017.

[19] X. Wang, L. Wang, and Q. Zeng, "Fractional differential equations with integral boundary conditions," Journal of Nonlinear Sciences and Applications, vol. 9, no. 4, pp. 309-314, 2015.

[20] J. Graef, L. Kong, Q. Kong, and M. Wang, "Uniqueness of positive solutions of fractional boundary value problems with non-homogeneous integral boundary conditions," Fractional Calculus and Applied Analysis, vol. 15, no. 3, pp. 509-528, 2012.

[21] X. Hao, L. Zhang, and L. Liu, "Positive solutions of higher order fractional integral boundary value problem with a parameter," Nonlinear Analysis: Modelling and Control, vol. 24, no. 2, pp. 210-223, 2019.

[22] X. Zhang, L. Liu, Y. Wu, and Y. Zou, "Existence and uniqueness of solutions for systems of fractional differential equations with Riemann-Stieltjes integral boundary condition," Advances in Difference Equations, vol. 2018, no. 1, Article ID 204, 2018 
[23] A. Ardjouni and A. Djoudi, "Positive solutions for nonlinear Caputo-Hadamard fractional differential equations with integral boundary conditions," Open Journal of Mathematical Analysis, vol. 3, no. 1, pp. 62-69, 2019.

[24] M. S. Abdo, H. A. Wahash, and S. K. Panchal, "Positive solution of a fractional differential equation with integral boundary conditions," Journal of Applied Mathematics and Computational Mechanics, vol. 17, no. 3, pp. 5-15, 2018.

[25] Z. Bai and T. Qiu, "Existence of positive solution for singular fractional differential equation," Applied Mathematics and Computation, vol. 215, no. 7, pp. 2761-2767, 2009.

[26] E. T. Zhi, X. P. Liu, and F. F. Li, "Existence of positive solutions for boundary value problems of fractional impulsive differential equation," Journal of Jilin University: Science Edition, vol. 52, no. 3, pp. 482-488, 2014. 